\title{
Impacto de la aplicación del aprendizaje cooperativo en aulas de Educación Básica
}

\author{
Gilberto Natividad Aranguren Peraza \\ gilberap@gmail.com \\ https://orcid.org/0000-0003-1480-9401 \\ Universidad Pedagógica Experimental Libertador (UPEL) \\ Caracas, Venezuela. \\ Marisol Sellet Zurita Aguilera \\ margasam4@gmail.com \\ https://orcid.org/0000-0002-2347-2575 \\ Universidad Nacional Experimental de las Fuerzas Armadas (UNEFA) \\ Caracas, Venezuela.
}

Recibido: 26/05/2021 Aceptado: 22/10/2021

\begin{abstract}
Resumen
El objetivo del artículo es describir el proceso de aplicación del aprendizaje cooperativo, a partir de las experiencias del profesorado en las aulas de clases de Educación Básica, en el marco de la necesidad de abordar estrategias y metodologías que satisfagan el desarrollo de experiencias educativas favorecedoras del aprendizaje, entendiendo que la cooperación beneficia tanto al estudiantado como al cuerpo docente y dadas las condiciones, tanto personales como institucionales, que limitan el surgimiento de experiencias innovadoras en la escuela. La información y los datos se obtuvieron durante el período escolar 2017 - 2018, mediante el uso de la Investigación - Acción. Se formularon categorías para el análisis y comprensión de los procesos surgidos durante la aplicación del aprendizaje cooperativo. La población estudiantil fue de 1018, siendo la muestra un total de 423, todo perteneciente a la Primera y Segunda Etapa de una Institución de la Escuela Básica, y el número de profesoras y profesores que participaron fue de 28. Entre los hallazgos se menciona que el aprendizaje cooperativo contribuye a mejorar las relaciones en el aula, su práctica facilita el desarrollo de habilidades cognitivas y sociales, siendo una conclusión de interés el que su aplicación orienta la enseñanza bajo un enfoque constructivista.
\end{abstract}

Palabras clave: Educación, Aprendizaje, Enseñanza, Formación, Cooperativismo.

\section{Impact of the application of cooperative learning in Basic Education classrooms}

\begin{abstract}
The objective of the article is to describe the process of application of cooperative learning, based on the experiences of teachers in Basic Education classrooms, within the framework of the need to address strategies and methodologies that satisfy the development of educational experiences that favor the learning, understanding that cooperation benefits both the student body and the teaching body and given the conditions, both personal and institutional, that limit the emergence of innovative experiences in school. The information and data were obtained during the school year 2017 - 2018, by using Research - Action. Categories were formulated for the analysis and understanding of the processes that emerged during the application of cooperative learning. The student population was 1018, the sample being a total of 423 , all
\end{abstract}


belonging to the First and Second Stage of Basic Education, and the number of teachers who participated was 28. Among the findings, it is mentioned that cooperative learning contributes to the improvement of relationships in the classroom, its practice facilitates the development of cognitive and social skills, being a conclusion of interest that its application facilitates teaching under a constructivist approach.

Keywords: Education, Learning, Teaching, Training, Cooperativism.

\section{Introducción}

Las escuelas se enfrentan a múltiples problemas socioculturales (Aranguren, 2017), que generan situaciones de índole emocional y sociocognitivo afectando las interacciones y el logro de los objetivos de aprendizaje. De manera, que la educación requiere de metodologías dinámicas que favorezcan la consolidación de habilidades cognitivas y sociales, así como la responsabilidad y la autonomía del estudiantado (Pamplona, Cuesta y Cano, 2019). Esto supone pensar en la práctica, formación y actualización del profesorado; por ello, la aplicación de estrategias favorecedoras de competencias sociales y cognitivas son imprescindibles en el fortalecimiento del aprendizaje (Morales, García, Torres y Lebrija, 2018; Collado y Fachelli, 2019) y en el desarrollo emocional del estudiantado (Goleman, 1996 y Bisquerra, 2000).

En este sentido, se hace necesario el desarrollo de destrezas y de herramientas teóricas metodológicas diferentes para actuar dentro de las aulas, por lo que se propone el aprendizaje cooperativo como estrategia alterna, dado su alcance en la adquisición de habilidades cognitivas mediante la interacción y socialización en equipos (Zurita, 2020), en el mejoramiento de las relaciones socioafectivas e interpersonales incrementando el valor de la responsabilidad y ayuda mutua (Morales, et. al., 2018).

Con base a estos supuestos, se desarrolló un proceso de intervención y acción en el aula basado en el aprendizaje cooperativo; por lo que un equipo docente de una institución escolar aplicó en sus aulas de clases estas estrategias para atender dos situaciones de interés: la primera, mejorar las condiciones socio cognitivas y afectiva del estudiantado y la segunda, favorecer un proceso de adquisición y procesamiento de herramientas orientadas al desarrollo del trabajo en equipo (Garrote, Jiménez y Martínez, 2019). En este sentido, se formularon las siguientes interrogantes a objeto de orientar el proceso de investigación: ¿Cuál es el alcance de la aplicación del aprendizaje cooperativo en el mejoramiento de la práctica docente? y ¿Cómo impacta la aplicación del aprendizaje cooperativo en la consolidación de rutinas favorecedoras del aprendizaje en estudiantes de primaria? 
Por lo que se planteó como objetivo el describir un proceso basado en la aplicación del aprendizaje cooperativo, a fin de comprender su alcance en el mejoramiento de la práctica y en la consolidación de rutinas favorecedoras del aprendizaje en estudiantes de educación básica, mediante el uso de la Investigación - Acción como método de investigación e intervención.

\section{El aprendizaje cooperativo}

El aprendizaje cooperativo es utilizado en educación como alternativa para la conducción del aprendizaje y la facilitación de la enseñanza (Villarrubia, 2016; Juárez, Rasskin y Mendo, 2019). Su aplicación permite que el estudiantado desarrolle competencias sociales y cognitivas (Trujillo, 2002; Zurita, 2020; Azorín, 2018), por estar sostenido en principios como el liderazgo distribuido, el docente como mediador, el agrupamiento heterogéneo, la interdependencia positiva, la adquisición de habilidades, la autonomía grupal y la ayuda mutua (Ferreiro y Espino, 2009; Muñoz y Cordero, 2017), los cuales conducen al progreso del aprendizaje.

Como modelo pedagógico - interactivo (Aranguren, 2018; Alarcón, et. al., 2018), el aprendizaje cooperativo busca el desarrollo del aprendizaje a partir de la socialización entre personas en un equipo. Johnson, Johnson y Holubec (1994) lo definen como una estrategia didáctica donde participan grupos reducidos de estudiantes, y cada uno trabaja para maximizar el aprendizaje propio y el de sus pares. Slavin (1999) señala que estas técnicas facilitan al estudiantado el trabajo en equipos heterogéneos. Por otra parte, Echeita (1995) señala que es un conjunto de actividades que asume el profesorado para impactar en el desarrollo de las habilidades cognitivas y sociales del estudiantado. En este caso, las estructuras de aprendizaje se orientan hacia el desarrollo de la autonomía, de la interdependencia, cooperatividad y responsabilidad.

Madrid, et al. (2013) definen el aprendizaje cooperativo como un enfoque para la construcción activa del conocimiento, mediante un conjunto de métodos que suponen la participación del profesorado y del alumnado. En este mismo sentido, Izquierdo, et al. (2019), señalan que el trabajo cooperativo se desarrolla de forma voluntaria y entre iguales, sin autoridad jerárquica que conduzca el proceso en los equipos, por lo que sus miembros comparten los mismos objetivos, y su trabajo es conducido mediante connotaciones democráticas, sin menoscabar que las decisiones generales en el aula están supeditadas a las condiciones establecidas por el docente desde principio del proceso. 
En los equipos el estudiantado experimenta relaciones horizontales facilitándole el reconocimiento de sus limitaciones y condiciones (Johnson y Johnson, 2004). Su aplicación permite el desarrollo del autoconcepto de sí mismo, comprendiendo y controlando sus emociones. A diferencia de otras metodologías, "se puede afirmar que en aquellos estudiantes que interactuaron cotidianamente en un escenario pedagógico cooperativo, se observó un aumento en el autoconcepto físico, personal, familiar y social" (Denegri, Opazo y Martínez, 2007, p. 33). Por ello, el profesorado requiere de herramientas metodológicas que conviertan al estudiantado en protagonista de su propio proceso de aprendizaje (Ceinos y García, 2009).

\section{Metodología}

El diseño de investigación utilizado es el cualitativo (Bautista, 2011), y el método es el de la Investigación - Acción; para ello se siguió la metodología señalada por Aranguren (2007), la cual permitió la reconstrucción de los escenarios a partir de las voces del personal docente y del alumnado, y del análisis de las informaciones aportadas. La experiencia se llevó a cabo durante el período escolar 2017 - 2018.

La escuela donde se desarrolló la experiencia fue una Unidad Educativa ubicada en una zona vulnerable al suroeste de la ciudad de Caracas, Venezuela. El estudiantado proviene de núcleos familiares señalados por el abandono del padre o de la madre o de ambos (Cuevas, 2010), ya sea por situaciones socio - afectivas o por la alta emigración producto del declive socio - económico experimentado en el país en los últimos años (Uzcátegui, Guzmán y Bravo, 2018). La comunidad, al igual que la escuela, se ven afectadas por una serie de carencias de servicios públicos como el agua, el transporte, electricidad, problemas de vialidad, entre otros, incidiendo, significativamente, en la asistencia a clases, en el ánimo y motivación, tanto del personal docente como del estudiantado.

La población estudiantil total fue de 1018 niños y niñas entre edades comprendidas entre 7 y 11 años respectivamente, trabajándose solo con el estudiantado de la Primera y Segunda Etapa de la Educación Básica, constituido por seis (6) grados con dos (2) secciones cada uno de ellos y conformado por 423 (41,55\%) estudiantes, de los cuales $220(52,01 \%)$ fueron del sexo masculino y 203 (47,99\%) del femenino. La distribución de los grupos se presenta en Tabla 1:

Tabla 1 - Distribución de los grupos de estudiantes por grados 


\begin{tabular}{cccc}
\hline Grado & Sexo Masculino & Sexo Femenino & Total \\
1 & 34 & 33 & 67 \\
2 & 37 & 34 & 71 \\
3 & 36 & 36 & 72 \\
4 & 41 & 29 & 70 \\
5 & 39 & 35 & 74 \\
6 & 33 & 36 & 69 \\
\hline Valores Absolutos & 220 & 203 & 423 \\
\hline Valores Relativos & 52,01 & 47,99 & 100 \\
\hline
\end{tabular}

En cuanto al personal docente, su número fue de 28: veinticuatro (24) docentes de aula, cuatro (4) de Apoyo Técnico, integrado por dos (2) Coordinadoras por turno, una (1) Coordinadora de Evaluación y Proyecto y un (1) Maestro de Educación Física, todos con más de dos años en sus cargos.

\section{Técnicas e Instrumentos de Recolección}

Las técnicas utilizadas para la recolección de la información fueron la Observación y la Entrevista: Las observaciones se aplicaron en las aulas de clases durante el desarrollo del aprendizaje cooperativo y en las reuniones con el personal docente. Para la recolección de la información se utilizó el Diario de Notas y Anécdotas que consistió en una libreta que sirvió para recoger de forma escrita los eventos suscitados durante las sesiones y en las visitas realizadas por los investigadores a las aulas de clases, recogiéndose detalles de los diálogos, observaciones sobre los métodos de aprendizaje cooperativo y de las reuniones con el equipo docente.

Las entrevistas se realizaron en dos modalidades, la primera fue la grupal, conocida como focus group (Valles, 1999), caracterizada, en este caso, por ser una conversación entre los investigadores y el equipo docente; la segunda versión fueron las individuales dirigidas a personas claves tanto del cuerpo docente como del alumnado, las mismas fueron seleccionadas tomando en cuenta su desempeño, participación e interés durante el proceso de investigación.

\section{Procesamiento de la información y de los datos}

El procedimiento para el análisis de los datos y de la información consistió en un sistema que involucró tres momentos específicos: la descripción de los hechos, el análisis de los mismos 
y la interpretación de los eventos (Zurita, 2020). Tanto la descripción como el análisis, producto de la información obtenida de los registros, permitió el surgimiento de las categorías dado que las descripciones fueron clasificadas y ordenadas en secuencias lógicas para visualizar los eventos y procesos dados, tanto en las aulas como en las reuniones con el cuerpo docente. La definición y descripción de las categorías surgió a partir de una serie de patrones presentes en los datos; esto permitió, mediante un examen de segmentos de textos, la clasificación de cada uno de ellos como evidencia y luego, debido a su recurrencia y saturación de contenidos, se crearon conceptos amplios que recogieran las nociones presentes en cada texto. Este proceso de análisis y teorización facilitó la interpretación (Pérez, 1994; Martínez, 1998), permitiendo la creación de nuevas relaciones teóricas para comprender el asunto en estudio. Por otra parte, se hizo uso de la negociación como mecanismo de legitimación y validación de la información y de sus interpretaciones. Para ello, se redactaron y leyeron actas de reuniones y se compartieron con los docentes las observaciones en aulas, así como sus análisis.

\section{Resultados e Interpretaciones}

Descripción del proceso

El trabajo siguiendo la secuencia: Exploración, Organización y Planificación de Actividades, Desarrollo y Sistematización.

\section{Exploración}

Consistió en la observación y descripción de las situaciones dadas en la escuela. Identificándose problemas puntuales tales como la falta de herramientas en el manejo de situaciones en el aula, la rutina reiterada de actividades repetitivas: "Todos los años tengo que escribir lo mismo en las planificaciones" - indicaba una docente - apreciación alentada también por otra: "Siempre se hace lo mismo, las clases son monótonas, los niños se fastidian, la Dirección del Plantel siempre quiere tener en sus manos la planificación, pero todos los años leen, sí es que lo leen, lo mismo". Para el personal docente las alternativas no eran suficientes, "no se había llegado a la raíz de los problemas": "Seguimos haciendo lo mismo, los problemas y sus tratamientos siguen siendo los mismos. Muchos cambios no se dan porque los docentes en verdad no cambiamos. Los problemas seguirán siendo los mismos hasta crear otras cosas con los niños. (Señalamiento realizado por una maestra).

Por otra parte, se logró percibir en el estudiantado situaciones de violencia y agresividad, evidenciado en peleas de corte agresivo y de índole vengativo. Siendo importante destacar que, 
durante los momentos de receso se revelaba una reiterada indisciplina estudiantil siendo corroborado por el profesorado durante las reuniones. Este conjunto de situaciones y condiciones condujeron a la formulación de un diagnóstico, planteándose la necesidad de un proyecto que brindara herramientas al docente para actuar en el aula mediante rutinas favorecedoras del aprendizaje y el mejoramiento de la convivencia escolar.

\section{Organización y planificación de actividades}

En virtud de lo anterior se propuso un proceso de acción basado en el aprendizaje cooperativo; siendo la propuesta elevada al Consejo Docente y acordándose en él, como actividades imprescindibles, la planificación y organización de las siguientes actividades: (a) talleres de conocimiento y manejo de las técnicas de aprendizaje cooperativo, con el objeto de brindar herramientas para la aplicación de los métodos en las aulas de clases; (b) aplicación del aprendizaje cooperativo y (c) reuniones periódicas para la socialización, valoración y sistematización de las actividades en el aula.

\section{Experimentación y Desarrollo de Acciones}

Este momento del proceso consistió en cuatro sistemas de actividades llevadas a cabo durante un lapso de nueve meses:

Actividades de adquisición y capacitación, que consistió en la capacitación del personal docente en la adquisición de herramientas sobre aprendizaje cooperativo; Actividades de ejecución, basadas en la aplicación del aprendizaje cooperativo en las aulas de clases por parte de los docentes, aplicándose los métodos Aprendiendo Juntos, El Rompecabeza, Investigación en grupos (Slavin, 1999) y Tutorías por pares iguales (Torrado, Manrique y Ayala, 2016), además de otros creados por el profesorado en sus aulas; Actividades de socialización, que fueron las reuniones dadas entre el equipo docente y el de investigación para socializar los avances y procesos llevados a cabo en las aulas de clases. Las reuniones se hicieron una vez al mes durante un período de siete meses y Actividades investigación e interacción que consistió en la realización de observaciones en el aula y de entrevistas tanto a miembros del personal docente como al estudiantado.

\section{Sistematización}


La sistematización de la experiencia se basó en la reflexión en equipo acerca de la intervención y aplicación del aprendizaje cooperativo en las aulas de clases; se llevó a cabo conjuntamente con el sistema de encuentros de socialización (Fernández, Barajas y Santillana, 2017). El proceso de sistematización se produjo con base a la información arrojada de las observaciones y entrevistas, haciendo uso de un análisis de categorías, para lo cual se realizaron matrices de análisis considerando los textos del Diario y de las entrevistas como evidencias. Durante la sistematización se revelaron situaciones que debían ser nuevamente planificadas y ejecutadas demostrándose con ello el carácter espiral de la Investigación - Acción.

\section{Categorías de análisis}

Fueron desarrolladas siete categorías de análisis (CA) a partir de la saturación de las evidencias descubiertas, estas categorías son las siguientes: CA1. Limitaciones del aprendizaje cooperativo en su aplicación; CA2. Flexibilidad del aprendizaje cooperativo; CA3. Desarrollo de habilidades lectoras y escritoras a partir de las interacciones grupales; CA4. La ayuda mutua; CA5. El aprendizaje cooperativo y el desarrollo de valores; CA6. Aplicación del aprendizaje cooperativo y el seguimiento de normas e instrucciones y CA7. Incidencias del aprendizaje cooperativo en la conducta.

\section{Análisis e interpretación de las categorías}

CA1. Limitaciones del aprendizaje cooperativo en su aplicación. Una de las limitaciones encontradas fue la dificultad de la aplicación del aprendizaje cooperativo en ambientes no acostumbrados a experiencias de aprendizaje grupal: "Al comienzo fue difícil, los niños no estaban acostumbrados a trabajar en grupo. Estuve guiándoles en caso de dificultades, hicieron uso del diccionario para ayudarse en las correcciones... Al comienzo algunos se mostraban rebeldes, se atacaban con violencia...” (Expresó una educadora). En cuanto a esto, una observación realizada en aula reveló lo siguiente:

La actividad de interacciones cooperativas consistió en agrupar a los estudiantes en equipos no mayores de cinco miembros. Los mismos elaboran sus informes y ayudados entre ellos construyen mapas. El estudiantado no está acostumbrado a trabajar en equipo. Nunca han desarrollado el sistema de interacciones, le es complicado, aun así, se nota que lo hacen muy bien. Para el momento de la construcción de los mapas se agrupan, conversan entre ellos y se percibe que discuten en torno al trabajo realizado. Se escucha que conversan acerca de otros aspectos. Hubo momentos de tensión cuando un estudiante saca constantemente el 
celular. Se nota que les cuestas estructurar el mapa, conversan entre ellos para elaborarlo y desarrollar la estrategia. No se preguntan acerca del progreso del trabajo. (Tomado del diario de Notas y Anécdotas del 180315).

Revelo, Collazos y Jiménez (2017), afirman que el estudiantado encuentra a menudo dificultades en actividades colaborativas y cooperativas al compartir recursos y discutir ideas, ya que estas prácticas rompen con los esquemas tradicionales, con la cual la mayor parte de la población estudiantil y del profesorado está acostumbrado.

Las nuevas condiciones del aula con la puesta en práctica del aprendizaje cooperativo condujeron, tanto al profesorado como al estudiantado, a reorganizar sus relaciones y modos de comunicación. El desorden, la confusión, el miedo a participar, la lucha por la obtención de los liderazgos internos en los equipos, las discusiones innecesarias, así como las peleas y los gritos fueron las escenas más comunes vistas durante los primeros momentos de conducción de estas estrategias, así lo revela la siguiente descripción de una observación realizada en un aula de clases:

Se observa mucho desorden y confusión. Resulta extraño para ellos la distribución del aula y las nuevas disposiciones de la maestra... los miembros de cada equipo discuten acerca del tema. Algunos se perciben confundidos. Uno de los equipos terminó antes de tiempo y trabajan con la exposición, juegan entre ellos. Una niña enfurecida grita a sus compañeros de equipo: ¡Ayúdenlo! - refiriéndose a la necesidad de ayudar a un joven que estaba haciendo una de las actividades encomendadas. Muchos estudiantes se acercan a la maestra para preguntarle cualquier cosa. Hay tensiones en los equipos. Muchas discusiones versan sobre cómo distribuir responsabilidades. Se alzan la voz entre ellos... En un equipo uno de sus miembros asume el liderazgo e intenta ayudar a sus compañeros haciéndoles preguntas, se observa que sus miembros participan muy poco. $\mathrm{Ni}$ siquiera hablan entre ellos. No quisieron presentar el material trabajado, uno de ellos dijo: - ¡Yo no voy hacer el ridículo! - la maestra intentó persuadirlo, invitándolo a participar, cosa que no hicieron... (Tomado del diario de Notas y Anécdotas del 180323).

En algunas ocasiones, debido a la indisciplina y violencia interna en el aula, las actividades se salían de la conducción del personal docente, arriesgándose de este modo los objetivos y metas propuestas al inicio:

Los estudiantes conformaron grupos de tres miembros; la idea era que investigaran en equipo y discutieran. Al final debían construir un mapa conceptual. La maestra intervino en un acto que podía convertirse en violencia. Dentro de los equipos sus miembros no se respetan, de hecho, entre ellos se lanzan los famosos "taquitos". Se escuchó varias veces a un equipo leer en voz alta. El grupo en general se veía trabajando y 
discutiendo. Algunos estudiantes se arrinconaron y se veían trabajando solos (Tomado del diario de Notas y Anécdotas del 180328).

Otra limitación fue el temor expresado por algunos miembros del personal docente, en cuanto a esto una maestra señaló lo siguiente: "Me sentía como perdida, pareciera que me hubiesen tocado todos los niños con problemas para que yo les diera clase", "era algo nuevo y no sabía qué hacer...". Para este personal abordar estas metodologías supuso un reto, porque las aulas cooperativas no responden al orden de las aulas tradicionales (Slavin, 1999). Por otra parte, una educadora llegó a expresar que su mayor dificultad fue observar cómo los estudiantes se distribuían en los equipos:

Con la aplicación de las estrategias de aprendizaje cooperativo se presentaron grupos selectos, grupos de niños y niñas que creían que lo sabían todo, y por supuesto discriminaban a otros. Estos grupos impedían que otros estudiantes integraran el equipo y discutieran, traté en lo posible de desintegrarlos de manera que otros pudiesen intervenir y de este modo aprovechar lo aprendido por todos. Logré separarlos e integrarlos en otros equipos, descubrí que el grupo en general realizó presiones para evitar que esto continuara ocurriendo.

Estas limitaciones no difieren de las encontradas por López (2015):

(...) el alumnado carece de hábitos de trabajo cooperativo; los equipos no saben cómo desarrollar la responsabilidad individual dentro del grupo ni cómo distribuir la tarea haciendo que todos desempeñen un papel al margen de contestar preguntas. Se verifica además una fuerte tendencia al individualismo y a la segregación (p.171).

Se destaca como una limitación la poca información que el personal docente tenía acerca de las estrategias de aprendizaje cooperativo, esto los mantuvo a la expectativa en relación con los efectos de los métodos en el estudiantado. La aplicación de las estrategias supuso una redefinición del papel del personal docente en las aulas (Ceinos y García, 2009), esto implica formarse en su aplicación para favorecer transformaciones de la práctica educativa y acceder, en el marco de su formación, a lo que sus colegas son capaces de sentir, pensar y hacer acerca de su praxis (León, et. al, 2011).

CA.2.- La flexibilidad del aprendizaje cooperativo. Se destacó la falta de rigidez del aprendizaje cooperativo, en este sentido, una maestra señaló: "No es un trabajo que requiere colocar a los estudiantes en situaciones rígidas, más bien la dinámica diaria con la estrategia pone al estudiante más relajado, pudiendo hablar, reír, caminar, interactuar con mayor facilidad con sus compañeros". La aplicación de estas metodologías supuso, entre otras cosas, preparar materiales y planificar dinámica estudiantil basada en la cooperación y la demostración 
de habilidades y destrezas sociales. Oberto (2014) señala que el aprendizaje cooperativo "es un modelo estructurado riguroso y exigente" (p. 68), por lo que su rigidez tiene que ver con el cómo se aplica para que facilite ambientes que permitan un trabajo sin presión y los equipos interactúen con mayor facilidad, posibilitando la adquisición de destrezas comunicativas como el "escuchar, ceder, argumentar, respetar turnos de participación, preguntar, responder" (León, et. al, 2011, p. 723).

La flexibilidad indicada se trata de la experiencia interna dada en los equipos, evidenciándose en señalamientos como: "porque me divierto mucho y aprendo", "en equipo podemos intercambiar ideas y discutir las preguntas", "discutimos juntos y nos explicamos mutuamente", "todos aportamos ideas", "muchos dan diferentes opiniones y se ponen más claras las preguntas", "podemos intercambiar ideas", nociones rescatadas de las entrevistas realizadas al alumnado, expresando sus estados de ánimo dentro de los equipos; para ellos, estas metodologías representan la oportunidad de aprender de un modo diferente, tal como lo señaló un estudiante: “Bueno, a mí me gustó mucho trabajar con mis compañeros en grupo, uno aprende un poco más y a veces nos divertimos mucho, claro siempre y cuando tengamos un límite para saber cuándo divertirse".

Toda flexibilidad dada durante la aplicación del aprendizaje cooperativo indica el desarrollo de competencias personales y sociales favorecedoras de la autonomía (Peña, 2010). Una de las características fundamentales del aprendizaje cooperativo es que posee reglas que la rigen, y en la medida que se implementan en el aula de clases, el estudiantado va adquiriendo hábitos y se van desarrollando los cambios: "cuando se aplica el aprendizaje cooperativo y los alumnos descubren que este sistema tiene reglas que son necesarias cumplirlas para alcanzar el nivel del equipo, entonces comienzan a verse los cambios en ellos” (Zurita, 2020, p. 67).

CA.3.- Desarrollo de habilidades lectoras y escritoras a partir de las interacciones grupales. Un énfasis del proceso experimentado fue enfrentar las dificultades de lectura y escritura. Las metas se orientaron a que el estudiantado pronunciara correctamente las palabras escritas, comprendiera e interpretara lo que leen, expresara con sus propias palabras lo leído y escribiera correctamente. El uso de cuentos y poesías fue imprescindible tanto para asumir la lectura y escritura como para discutir, reflexionar y comprender el significado de los valores de la paz y la no - violencia. Una maestra señaló que condujo a sus estudiantes a que se convirtieran en correctores de sus compañeros, expresando, de forma entusiasta, que: "mejoraron la 
redacción, ahora lo hacen más coherentemente, los trabajos grupales mejoran la calidad de los trabajos presentados".

Para el personal docente una meta importante fue la de lograr que el estudiantado se iniciara en el proceso lector y escritor; esto permitió que los trabajos siguientes se realizaran con mayor facilidad. La iniciación en el proceso lector implicó un esfuerzo de motivación que se llevó a cabo mediante el uso de textos cortos, pero que reflejaran un contenido trascendental para ser discutido. Siendo un logro significativo lo siguiente: “Fue un año irregular, al final quedé con 26 estudiantes, hubo 9 que no lograron aprender a leer, creo que hace falta atenderlos más... mi trabajo de aprendizaje cooperativo lo basé en la lectura y la escritura, y en los valores" - señaló una docente. Bajo este mismo tenor, otra educadora señaló lo siguiente: “Lo más importante fue la motivación a leer, es tal vez la fase más difícil, pero al lograrlo, lo demás es más fácil, yo creo que todos mis estudiantes aprendieron a leer, unos mejores que otros, pero aprendieron..."; de igual modo, otra maestra señalaba:

Aunque fue difícil, los muchachos aprenden unos de otros, las tutorías por pares enseñan eso. El proceso de corrección entre ellos los somete a asumir sus errores y a aprender de ellos mismos. La estrategia de tutoría por pares del aprendizaje cooperativo ayudó a muchos a que profundizaran en la comprensión lectora.

Por otra parte, el uso de cuentos relacionados con la paz y la no - violencia, facilitó la discusión de la necesidad de una mejor convivencia escolar, con relación a esto una maestra señaló lo siguiente:

Cuando se discutía acerca de la paz y la no-violencia, los muchachos demostraban la necesidad de vivir en una sociedad más justa. El barrio donde viven no ofrece la seguridad que ellos quieren y quieren sus padres. Señalaban que era importante que la gente viviera unida y se quisieran unos a los otros.

En este segmento se presentan dos situaciones, la primera es la aplicación del aprendizaje cooperativo en la enseñanza de la lectura y la escritura, y la segunda es la reflexión que esto supone acerca de la paz y la no violencia como fórmula de convivencia escolar. Con respecto a la primera situación, Trujillo (2002) señala que el aprendizaje cooperativo es una herramienta eficaz para la didáctica relacionada a la enseñanza de la lectura, porque las interacciones activan la comunicación generando procesos de desarrollo cognitivos; al igual, Cassany (2004), señala que el desarrollo del diálogo en equipos facilita el lenguaje escrito por su eficacia en la regulación de los procesos de composición que llevan a cabo los estudiantes dentro de los grupos. En los equipos cooperativos las estructuras cognitivas previas que tiene el estudiante le 
permite hacer relaciones con la nueva información que obtiene durante las interacciones generando mayor retención y haciendo significativo el aprendizaje, "al estar claro en la estructura cognitiva, se facilita la retención del nuevo contenido y, en consecuencia, la nueva información se relaciona con la anterior y se guarda en la memoria a largo plazo (Arroyo, et. al, 2010, p. 39).

Por otra parte, para muchos miembros del personal docente le era complicado hacer chequeos de lecturas, ya que los procedimientos utilizados no funcionaban del todo bien. Generalmente, estas prácticas en el aula incomodan a los estudiantes. Por ello, una docente describió una técnica basada en el aprendizaje cooperativo la cual aplicó en su aula a objeto de facilitar esta actividad:

El trabajo cooperativo se desarrolló durante dos jornadas: en la primera los estudiantes realizaron una lectura de forma silenciosa, enumeraron los párrafos y elaboraron con cada párrafo una idea, para luego construir con todas las ideas un texto más grande. En la segunda jornada: conversé con ellos acerca de la importancia de la ayuda mutua y la responsabilidad en los equipos. Los equipos estaban constituidos por tres estudiantes, la idea era que discutieran entre ellos el texto elaborado por cada uno el cual lo resguardé con sus nombres. Posteriormente, los equipos se desintegraron y conformaron grupos más grandes (de cinco miembros), conformados al azar, en estos equipos discutieron el tema y ampliaron el contenido. Después de un tiempo de trabajo de discusión se conformaron de nuevo los equipos originales, o sea los tres que se integraron al inicio, y construyeron entre ellos un mapa mental con los textos construidos.

En esta secuencia de actividades programadas se visualizan dos aspectos: el primero, es que el estudiantado desarrolló trabajos en forma individual; elaboraron en el aula materiales para socializar, garantizando los recursos para interactuar en los equipos. El segundo aspecto, fue que los estudiantes discutieron en equipos pequeños a partir de sus construcciones para luego socializar lo analizado con equipos más grandes. La participación en estos niveles de agrupación ayuda a nutrir al estudiantado en los contenidos que se desarrollan y les permite su revisión desde diversas ópticas. Este procedimiento contribuye al desarrollo de los conflictos socio cognitivos (Medrano, 1995), pero también permite la evidencia de la Zona de Desarrollo Próximo (ZDP) planteada por Vygotsky (1979), que establece que durante la acción de los estudiantes en la zona "las interacciones estarán dominadas por las comprensiones de la persona más experimentada, que puede ser el maestro" (Labarrere, 2016), oportunidad que puede ser desempeñada por cualquiera de los estudiantes en los equipos. 
Por otra parte, se observó a una maestra aplicar el aprendizaje cooperativo para construir textos escritos, la experiencia se desarrolló en el 6to grado, en el área de Ciencias Sociales:

Al inicio se dividió el grupo general en equipos de tres. A cada equipo le hizo entrega de un papel que contenía un título del tema. Todos los estudiantes elaboraron una composición a partir del título entregado al grupo y luego lo leyeron en voz alta a sus compañeros. El docente escribió en el pizarrón una serie de interrogantes que debían ser respondidas en la medida que se escuchaban las composiciones en cada grupo. Las preguntas fueron las siguientes: ¿Es una lectura agradable? ¿Qué es lo que no te gustó de la lectura? ¿Qué mejorarías tú a lo escrito por tu compañero? ¿Crees que, lo expresado por tu compañero en la composición, es cierto o es falso y por qué? Después de las lecturas de los textos y de las retroalimentaciones, los estudiantes pasaron el material escrito a su compañero de la derecha para que lo valoraran en cuanto a la ortografía y redacción, y así sucesivamente hasta que el material llegó nuevamente a su autor. Después de esto cada estudiante elaboró nuevamente su texto, volviéndolo a leer en voz alta frente a sus compañeros. Después de este proceso cada equipo redactó un documento que fue expuesto en voz alta ante todo el curso. Se observó que la docente atendió a todos los grupos, interviniendo en el desarrollo interno de cada uno de los equipos. La evaluación fue grupal e individual; la maestra solicitó el material elaborado por cada uno de los estudiantes para su revisión y la evaluación grupal correspondió a la elaboración en equipo del material escrito al final, así como la discusión y las observaciones finales (Tomado del diario de Notas y Anécdotas del 180531).

En esta experiencia se pudieron observar varias situaciones de interés: (a) el ritmo del trabajo se llevó a cabo mediante una secuencia que supuso el trabajo individual, luego grupal, hasta hacerlo común a toda la comunidad del aula; (b) la presencia de la maestra en los equipos es fundamental para el logro de las competencias y objetivos; (c) se evidencia que la escritura puede convertirse en una construcción colectiva; (d) se hizo uso de los contenidos de sociales, pero el énfasis era la adquisición de habilidades y destrezas para la escritura, pudiéndose aplicar con cualquier otro contenido y (e) la posibilidad de convertir al estudiantado en expertos, creándose condiciones en los equipos para que aprendan a justificar los contenidos.

CA.4.- La ayuda mutua. La puesta en práctica del aprendizaje cooperativo determina situaciones que contribuyen al logro de metas, en cuanto a esto una maestra señalaba lo siguiente:

Ha permitido que los estudiantes se integren en equipo y se motiven a la investigación, desarrollan trabajos escritos y manuales con mayor facilidad, además se familiarizan con la lectura a través de los cuentos y de las redacciones hechas por ellos mismos, de esa manera expresan sus 
ideas y sus pensamientos. En cuanto a las matemáticas trabajan con materiales concretos, han desarrollado destrezas y habilidades en las operaciones básicas y de lógica.

La aplicación de estas estrategias facilita procesos de enseñanza bajo un enfoque constructivista y colectivo: "Entre los resultados de suma importancia, está la integración de los estudiantes, ellos construyen conocimientos. Los mismos niños se corrigen" (Afirmó una docente). Esta integración del estudiantado genera aprendizaje con base en el apoyo mutuo (Slavin, 1999), ya que mediante estos métodos el proceso de aprender se sostiene en el apoyo entre los pares. El fortalecimiento del principio de ayuda mutua, mediante las correcciones y observaciones entre estudiantes, redunda en la formación de personas más responsables y con un nivel de participación más alto: "La tarea fue dura, pero logré la integración, sentí que los estudiantes aprendieron juntos. Ahora socializan con facilidad entre ellos y conmigo. Comprobé que si trabajan en casa... además, las calificaciones aumentaron... - llegó a expresar una educadora. Situaciones como estas facilitan que el estudiantado decida con un mayor sentido de responsabilidad, y su autoestima se eleva a partir del reconocimiento, por parte del otro, de su proceso de aprendizaje.

Para el alumnado el aprendizaje cooperativo resultó interesante: "Yo le voy a decir que el trabajo en grupo fue muy bueno porque todos nos ayudamos..." - afirmaba un estudiante. Con el trabajo en equipo el estudiantado merma sus conflictos, busca posibilidades de fortalecer sus relaciones humanas y encuentran el equilibrio en sus interacciones. Por supuesto, esto se concibe en torno a la necesidad de "dejar las diferencias" como principio básico para que el estudiantado se ayude mutuamente: "Me parece que con los trabajos en equipos nos hemos unido mucho más que el año pasado, hemos aprendido a dejar las diferencias y ayudarnos los unos a los otros, yo me desenvuelvo mejor con mis compañeros - decía una estudiante. El acompañamiento mutuo ayuda, porque en él se estructura el sentido de la confianza que genera equilibrio en el grupo, y contribuye que sus miembros no se sientan desamparados.

CA.5.- El aprendizaje cooperativo y el desarrollo de valores. La discusión acerca de la profundización de los valores en el aula tuvo como preámbulo la descripción de las evidencias de las actitudes que afectaban las relaciones entre el personal docente y el alumnado. Considerando este análisis, el personal docente señaló la necesidad de armonizar las relaciones entre los docentes y el estudiantado, a fin de propiciar espacios que le ayuden a diluir sus conflictos y revisar las actitudes para su aprovechamiento y reflexión; de igual modo se acordó 
que las relaciones entre los docentes debían expresar armonía de modo que el estudiantado tomara dicha experiencia como un patrón referencial en sus relaciones.

Por otra parte, una docente describió que su experiencia con aprendizaje cooperativo sirvió para establecer una discusión y análisis de los valores: "A partir de los trabajos cooperativos, logré rescatar los valores de honestidad, respeto, responsabilidad y amistad. Los muchachos desarrollaron capacidad para trabajar en equipo y para tolerarse mutuamente". El aprendizaje cooperativo contribuyó a fortalecer el encuentro grupal y a valorar actitudes demostradas por el estudiantado como la amistad, la responsabilidad y el respeto entre ellos. Una forma de experimentar la responsabilidad y reflexionar con los niños y niñas su importancia, fue la valoración del aseo personal como un elemento de proyección de la salud y el respeto por el cuerpo, al respecto una docente señaló:

Discutía mucho con mis alumnos porque a veces llegaban sin bañarse, sin ni siquiera con los dientes cepillados, me costó mucho hacerles entender la importancia que tiene el asearse todos los días, en esto la reunión grupal contribuyó mucho, los muchachos con su sinceridad se decían las cosas tal cual como la sentían...

En este sentido, una educadora señalaba que una forma de promover la responsabilidad del estudiantado en el aula era dejándolos solos por un período prudencial:

Al principio era terrible el dejarlos solos, originaban peleas, discusiones, gritos. Me llamaron la atención del por qué los dejaba solos. Después venía la discusión con ellos acerca de la necesidad que realizaran los trabajos en el aula sin mi vigilancia permanente, que asumieran su responsabilidad.... al final los muchachos pueden trabajar solos, me ausento por minutos y el salón permanece tal cual como lo dejé, los muchachos trabajan con mayor responsabilidad.

Durante el proceso de discusión acerca del significado del valor de la responsabilidad, miembros del personal docente apoyaron el uso de las sanciones como forma de reforzar el valor. Esto supuso una discusión que concluyó en que la responsabilidad debe ser promovida en el aula a objeto de que el estudiantado demuestre actitudes de autocontrol, disciplina y reflexión sobre sus acciones, y que ninguna acción debería ser abordada - con base al miedo y a la presión - siendo necesario profundizar aún más su importancia en la construcción de nuevas fórmulas.

Por otra parte, una educadora llegó a señalar que la estrategia de "ayuda entre pares iguales" permitió un mayor conocimiento en el estudiantado; el hecho que algunos permitiesen que otros los ayudaran era un "síntoma de que las relaciones entre ellos estaba mejorando", a su vez, señalaba que "esta experiencia promovió en el aula el valor de la ayuda mutua, los niños 
que sabían más ayudaban a aquellos que no sabían... entre ellos se criticaban el trabajo, a su vez aprendieron a respetar las cosas que hacían”- señalaba.

Vale la pena considerar la importancia de la reflexión como un valor del aprendizaje cooperativo, en tal sentido, una de las educadoras señalaba que estas metodologías permitieron convertir el aula en un espacio reflexivo: "percibo equipos que discuten y reflexionan en su proceso de construcción de los materiales". Siendo importante destacar que el profesorado debe favorecer, durante la aplicación del aprendizaje cooperativo, no solo la capacidad de pensar, sino también la reflexión en un espacio de confianza (Guerra, Rodríguez y Artiles, 2019). Otra educadora indicaba que la reflexión le fue útil en el manejo del valor de la responsabilidad, evidenciándose en "la entrega puntual de los trabajos, la asistencia a clase y la realización de las tareas". Hay que destacar que la reflexión, como ejercicio de aprendizaje, permite que la persona tome conciencia en cuanto a su forma de pensar y actuar (Pérez, et. al, 2015), de modo que esta práctica conduce al estudiantado a un nivel significativo de la comprensión de sus actuaciones.

Por último, hay que considerar que uno de los principios del aprendizaje cooperativo es el desarrollo de las habilidades sociales, porque permiten que el docente gestione los conflictos dentro del aula (Moya y Zariquiey, 2008). El debate que se desarrolla dentro de los equipos cooperativos, más allá de crear y profundizar las situaciones conflictivas, facilitó la regulación de las relaciones y emociones, permitiendo la comprensión de los problemas, así como la resolución pacífica de los conflictos internos que se originan producto de las interacciones; de manera, que el trabajo en equipo cooperativo incidió en la disminución de la violencia y las agresiones entre los estudiantes.

Para el personal docente fue importante dirigir un proceso mediante los valores de la paz y la no-violencia, noción que facilitó la experimentación de otros valores como la justicia, solidaridad, ayuda mutua, humildad, participación, respeto a la diferencia, la autoestima, la integración, la libertad, la verdad, la responsabilidad y la crítica como un medio para aceptar y comprender las dificultades. Hay que destacar que el personal docente enfatizó en que la violencia, la agresividad, la discriminación, el egoísmo, la mentira y la irresponsabilidad, representaban los valores de la vida cotidiana del estudiantado, por lo que era "necesario generar situaciones que pudieran romper con esa forma de vida. 
CA.6.- Aplicación del aprendizaje cooperativo y el seguimiento de normas e instrucciones. La aplicación del aprendizaje cooperativo permitió que las discusiones y los trabajos en los equipos se realizaran siguiendo normas:

La maestra previamente dictó las normas, los equipos se conformaron con tres miembros cada uno con una asignación dada por ella. El primero elabora el informe, el segundo prepara una breve exposición y el tercero construye un mapa conceptual o mental. Después cada quien elabora su material y lo ponen en común en el equipo. (Tomado del diario de Notas y Anécdotas del 1800607).

El aprendizaje cooperativo requiere de normas concretas que faciliten el desenvolvimiento interno de los equipos. Cada miembro es responsable de lo que le corresponde internamente en el grupo; juntos trabajan con responsabilidades sabiendo que del éxito de su trabajo depende la evaluación definitiva de todos los integrantes (Vaillant y Manso, 2019). A este respecto, una de las educadoras señaló: "durante el desarrollo de las interacciones aprendieron a respetar las oportunidades y los momentos de cada equipo y de cada compañero". Siendo necesario destacar que los trabajos grupales se desarrollaron siguiendo la dinámica propia de cada equipo. Los grupos establecieron sus propias reglas, en algunos casos hasta sus propias formas de sanción y recompensas:

Los estudiantes comienzan a organizarse entre ellos, discuten, se organizan, se gritan. Expresan en qué actividades puede desempeñarse, se enfrascan en discusiones acerca de cuáles son las responsabilidades de cada quien. Al principio el grupo en general se convierte en un desorden, que va cambiando, en la medida que los estudiantes se tranquilizan y van asumiendo las responsabilidades asignadas (Tomado del diario de Notas y Anécdotas del 180220).

La metodología incide, significativamente, en el desarrollo de habilidades sociales, permitiéndoles al estudiantado la posibilidad de crear acuerdos y asumir sus responsabilidades durante la ejecución de las tareas asignadas. Las discusiones y conflictos son resueltos sin que exista violencia, así se aprende a mejorar actitudes y a establecer compromisos internos en los equipos. El uso de normas internas se logra mediante el desarrollo de habilidades interpersonales y grupales, permitiendo al estudiantado aprender a comunicarse, a organizarse, a tomar decisiones consensuadas y a valorar sus relaciones. "Todo ello se hace a través de la enseñanza - aprendizaje de técnicas de escucha activa, de participación y de debate” (Azorín, 2018, p. 186).

El seguimiento de instrucciones y el acompañamiento del docente permiten que las tareas se logren con satisfacción: "hicimos lo que la maestra nos decía que teníamos que hacer", 
"hicimos el trabajo que la maestra nos dijo que hiciéramos", "la maestra siempre estuvo con nosotros, ayudándonos" - fueron afirmaciones de algunos de los estudiantes. El alcance de los objetivos en el aprendizaje cooperativo es posible mediante el seguimiento de instrucciones, el docente es la persona más indicada para darlas y orientar las prácticas en los equipos. Las instrucciones ayudan a la interdependencia positiva, por lo que el docente debe ser explícito al indicarlas, de manera que se comprenda que el esfuerzo de cada miembro del equipo facilite el aprendizaje de todos, ya que la "interdependencia positiva crea un compromiso con el éxito de otras personas, además del propio, lo cual es la base del aprendizaje cooperativo" (Johnson, Johnson y Holubec, 1999, p. 21).

CA.7.- Incidencias del aprendizaje cooperativo en la conducta. En diversas oportunidades el personal docente llegó a señalar la necesidad de que los estudiantes desarrollaran una mejor conducta en el aula: "algunos muchachos pasan la mañana sólo molestando a sus compañeros, pareciera que les faltara concentración y mayor atención en las cosas que hacen"- señala una educadora. La discusión en el equipo docente se basó en la necesidad de aprender a detectar situaciones conductuales, una maestra señalaba las dificultades que tienen para detectar trastornos o condiciones específicas: "Como maestra me siento desarmada, no tenemos herramientas que nos ayuden a diferenciar cuándo un niño que entra en nuestras aulas tiene problemas que deban ser revisados por especialistas". Esto condujo al equipo docente a discutir algunas pautas de interés a fin de favorecer la ayuda al estudiantado en conjunto con un equipo especializado de manera que se realizaran evaluaciones pertinentes y se les diera la atención adecuada, al igual se les ayudara al docente con herramientas y recomendaciones.

Durante una de las experiencias de aplicación del aprendizaje cooperativo pudo observarse la siguiente situación:

(...) los estudiantes tienden a gritarse y maltratarse. La maestra se disgustó debido a que una alumna encendía su teléfono y colocaba música a todo volumen, solicitó que el teléfono fuese apagado y la niña lo hacía pero al rato regresaba a encenderlo nuevamente. La docente se le acercó y le habló muy fuerte, señalándole que si continuaba colocando la música tendría que entregarle el celular ya que los otros equipos se inquietaban. Por otro lado, un niño llama idiota a otro de sus compañeros y le grita: bruto. (Tomado del diario de Notas y Anécdotas del 180220).

Situaciones como estas se revelaron con frecuencia en los grupos, sus miembros peleaban, se disgustaban y procuraban discutir y excluirse unos a otros ya sea por sus 
comentarios que tendían a ser crueles o por sus actitudes ofensivas; por ello, la necesidad de mecanismos que ayuden al profesorado a controlar más las aulas. En este sentido, una maestra explicaba cómo estas estrategias podían contribuir al proceso de regulación: "los mismos equipos tenían normas internas, en muchas ocasiones las normas no permitían que los niños demostraran conductas desagradables, muchas conductas mejoraron, muchas formas de comportarse fueron cambiadas debido a las relaciones que entre ellos se daban, los equipos se convirtieron en reguladores...". Para Johnson, Johnson y Holubec (1999), estas actividades suponen un modo de actuar y para ello es necesaria la creación de condiciones por parte del personal docente, por lo que éste debe ser explícito en sus instrucciones, haciendo énfasis en las conductas deseables para que las regulaciones se den en beneficio del estudiantado.

Por otra parte, la experiencia de otra profesional indicaba que la regulación de la conducta podía manejarse desde las relaciones suscitadas en los equipos:

Mi preocupación por la conducta me llevó a trabajar diversas estrategias, pasando por la sanción, al principio las estrategias de aprendizaje cooperativo me trajeron desagradables sorpresas, ya que los niños no estaban acostumbrados a trabajar en equipo, peleaban mucho y algunos aprovechaban para hacer de las suyas... después de varias semanas la situación se fue acoplando, comenzaron a trabajar más, aquello de "conductas difíciles" tuvieron que asumir responsablemente el trabajo...

Otra educadora señalaba que desde su percepción las conductas disruptivas del estudiantado se debían a lo aburrido que eran muchas actividades, a su modo de ver, la falta de atención no tenía nada que ver con el síndrome de déficit de atención e hiperactividad, pero que sí era importante el estar pendiente de las acciones de los niños para identificar esas dificultades y darle respuesta; en este sentido, vale la pena destacar una reflexión realizada por un miembro del personal docente:

Cuando llegué a aquí me sentía muy atrapada con eso de la conducta de los estudiantes. En verdad la mayoría se portan muy mal, les cuesta recibir órdenes, no siguen instrucciones y eso cuesta mucho. Al principio me costaba mucho llevarlos adelante, entre los gritos de ellos y los míos se iba la mañana. Muchos han sido los días en que me he ido a mi casa malhumorada. Cuando me propusieron estos métodos de trabajo sentía que estaba perdiendo el tiempo, pero cuando lo fui aplicando comencé a tomar las riendas del aula, me sentía más segura de mí misma y lo mejor era que ya no les gritaba a los estudiantes.

Esta experiencia revela el alcance de esta metodología, que no solo incide en la población estudiantil, sino también en los cambios de actitud y de conducta de los mismos educadores, mejorando su práctica y fortaleciendo su proceso de aprendizaje y comunicación con el entorno, 
dado que "es una metodología que favorece el desarrollo de competencias necesarias durante el proceso de formación y para el desarrollo laboral, como la comunicación activa, la responsabilidad o la autonomía” (Garrote, et al., 2019, p. 54).

\section{Conclusiones y recomendaciones}

En atención a lo anterior se presentan las siguientes conclusiones:

La aplicación del aprendizaje cooperativo en el aula permitió al estudiantado compartir y ayudarse mutuamente. Afirmación que responde a los principios que rigen a estas estrategias (García, 2018), los cuales están sostenidos en el desarrollo de las competencias sociales. Estas experiencias representan un sistema que provoca el aprendizaje mediante el compartir y las interacciones dadas durante los eventos que se desarrollan dentro de los equipos. Por lo que la aplicación del aprendizaje cooperativo facilitó procesos de enseñanza bajo un enfoque constructivista y colectivo.

Esta metodología influyó en el desarrollo de las habilidades sociales. El estudiantado pudo lograr acuerdos y asumir responsabilidades en la ejecución de sus tareas. En este sentido, los conflictos dados en los equipos pudieron ser resueltos sin violencia y agresividad, de esta manera el estudiantado aprendió a mejorar sus actitudes mediante un sistema de regulación y control de la conducta.

Entre las ventajas observadas durante la aplicación del aprendizaje cooperativo se señalan las siguientes: ayuda al mejoramiento de la capacidad comprensiva, contribuye al desarrollo de las competencias cognitivas y sociales del estudiantado, genera la lectura y discusión en equipo de materiales de interés, contribuye a profundizar el valor del respeto hacía sí mismo y hacia los otros, así como en la mediación de los conflictos, permite que el estudiantado experimente la escucha atenta a lo que expresan sus compañeros y ayuda a fortalecer lazos de amistad creando ambientes ricos en discusiones y en relaciones afectivas.

El proceso experimentado por estos docentes les permitió vincular y contrastar aspectos teóricos con la práctica diaria en las aulas, facilitando la comprensión de las posibles contradicciones, sobre todo aquella premisa inicial de creer que la investigación por sí sola soluciona el problema. En este sentido, fue necesario que el personal docente asumiera la investigación como una estrategia natural de trabajo, de acción e intervención que permite hacer lecturas e interpretación de las realidades existentes en las aulas. 
Entre las limitaciones que se evidenciaron se mencionan las siguientes: (a) las dificultades demostradas por el cuerpo docente para describir sus experiencias tanto de forma escrita como oral y el acceso a la institución debido a las dificultades de transporte y de recursos dada la situación político - económica del país.

Se recomienda evitar que el estudiantado se agrupe a partir de sus criterios e intereses personales, la organización en grupos heterogéneos facilita el desarrollo de los conflictos sociocognitivos y discusiones con mayor riqueza. El diseño de los trabajos en equipo debe permitir que cada uno de sus miembros tenga una actividad específica, de manera que el conjunto de todas las tareas internas se convierta en el producto esperado.

\section{Referencias}

ALARCÓN, E., Sepúlveda, P. y Madrid, D. (2018). Qué es y qué no es aprendizaje cooperativo. ENSAYOS. Revista de la Facultad de Educación de Albacete. $(33$ - 1) 205 - 220. Disponible: https://www.revista.uclm.es/index.php/ensayos/article/view/1575/pdf

ARANGUREN, P. G. (2007). La investigación - acción sistematizadora como estrategia de intervención y formación del docente en su rol de investigador. Revista Pedagogía. 28 (82) 173 - 195. http://www.redalyc.org/articulo.oa? id $=65908202$

ARANGUREN, P. G. (2017). La escuela como opción y espacio. Un análisis desde las concepciones y creencias. Areté: Revista Digital del Doctorado en Educación de la Universidad Central de Venezuela. 3 (5) 83 - 111. Recuperado: https://dialnet.unirioja.es/servlet/articulo?codigo $=6329696$

ARANGUREN, P. G. (2018). La estrategia verbal de enseñanza y el aprendizaje cooperativo integrado con películas de ciencia ficción. Revista Pedagogía. 39 (104) 9 - 29. Disponible: http://saber.ucv.ve/ojs/index.php/rev_ped/article/view/15696/0

ARROYO, S. M.; Faz de los Santos, L.; Gasca, G. G. y Orozco, C. R. (2010). Mejoramiento de la comprensión lectora basada en el aprendizaje colaborativo en la enseñanza media básica. Apertura. Revista de Innovación Educativa. 2 (2) 34 - 47. Disponible: http://www.udgvirtual.udg.mx/apertura/index.php/apertura/article/view/140

AZORÍN, C. (2018). El método de aprendizaje cooperativo y su aplicación en las aulas. Perfiles $\begin{array}{llllll}\text { Educativos. } & 40 & \text { (161) } & 181 & - & \text { 194. }\end{array}$ https://www.iisue.unam.mx/perfiles/numeros/2018/161

BISQUERRA, - A. R. (2000). Educación emocional y bienestar. Barcelona: Praxis.

BAUTISTA, N. (2011). Proceso de investigación cualitativa: epistemología, metodologías y aplicaciones. Bogotá: El Manual Moderno.

CASSANY, D. (2004). Explorando las necesidades actuales de comprensión. Aproximaciones a la comprensión crítica. Lectura y Vida.25 (2) 6 - 23. Disponible: $\underline{\text { https: } / / n 9 . c l / 1 m o 2 x}$

CEINOS, C. y García, R. (2009). El aprendizaje cooperativo como propuesta de innovación en el proceso de enseñanza -aprendizaje: Descripción de una experiencia. Actas do $X$ Congresso Internacional Galego - Português de Psicopedagogia. Braga: Universidade do Minho. $\quad 969 \quad$ - $\quad 979 . \quad$ Disponible: http://www.educacion.udc.es/grupos/gipdae/documentos/congreso/xcongreso/pdfs/t3/t $\underline{3 c 67 . p d f}$ 
COLLADO, S. A. y Fachelli, S. (2019). La competencia de trabajo en equipo: una experiencia de implementación y evaluación en un contexto universitario. REIRE, Revista d'innovació $i$ Recerca en Educació. $12 \quad$ (2) 1 - 21. https://ddd.uab.cat/pub/artpub/2019/203922/reire_a2019v12n2m07iSPA.pdf

CUEVAS, H. A. J. (2010). Jefas de familia sin pareja: estigma social y autopercepción. Estudios Sociológicos. $\quad 28 \quad$ (84) $753 \quad-\quad$ 789. Disponible: https://dialnet.unirioja.es/descarga/articulo/6164430.pdf

DENEGRI, M. O. C. y Martínez, G. (2007). Aprendizaje Cooperativo y desarrollo del autoconcepto en estudiantes chilenos. Revista Pedagogía 28 (81) 13 - 41.

ECHEITA, S. G. (1995). El aprendizaje cooperativo. Un análisis de sus ventajas respecto a otras estructuras de aprendizaje. En: Fernández, Pablo y Melero, Ma. Ángeles (comps.): La interacción social en contextos educativos. (pp. 167 - 186). Madrid: Siglo XXI España Editores.

FERNÁNDEZ, J. B. G. y Santillana, H. (2017, noviembre). Sistematización de experiencias en la formación de doctorantes, una metodología para la mejora docente. Ponencia presentada en el XIV Congreso Nacional de Investigación COMIE. San Luis de Postosí, México.

FERREIRO, G. R. y Espino, C. M. (2009). El ABC del aprendizaje cooperativo: Trabajo en equipo para enseñar y aprender. (2da. edic.) México: Trillas.

GARCÍA, V. M. C. (2018). Competencias clave y aprendizaje cooperativo, conceptos fundamentales en la educación actual. Revista International Studies on Law and Education . 29/30 79 - 90. Disponible: http://rua.ua.es/dspace/handle/10045/72368

GARROTE, D.; Jiménez, F. S. y Martínez, H. N. (2019). El Trabajo Cooperativo como Herramienta Formativa en los Estudiantes Universitarios. REICE. Revista Iberoamericana sobre Calidad, Eficacia y Cambio en Educación. 17 (3) 41 - 58. https://doi.org/10.15366/reice2019.17.3.003

GOLEMAN, D. (1996). Inteligencia emocional. Madrid: Kairós.

GUERRA, M. R. J. y Artiles, J. (2019). Aprendizaje colaborativo: Experiencia innovadora en el alumnado universitario. REXE. Revista de Estudios y Experiencias en Educación. 18 (36). 1 - 16. Disponible: http://www.rexe.cl/ojournal/index.php/rexe/article/view/661

IZQUIERDO, R. T., Ascencio. E.; Escarbajal, A. y Rodríguez, J. (2019). El aprendizaje cooperativo en la formación de maestros de Educación Primaria. Revista de Investigación Educativa. $37 \quad$ (2), 543-559. Disponible: https://revistas.um.es/rie/article/view/369731

JUÁREZ, P. María.; Rasskin, G. I. y Mendo, S. (2019). El aprendizaje cooperativo, una metodología activa para la educación del siglo XXI: Una breve revisión bibliográfica. Revista Prisma Social. $26200 \quad-\quad 210 . \quad$ Disponible: https://revistaprismasocial.es/article/view/2693

JOHNSON, D. y Johnson, R. (2004). Cómo reducir la violencia en las escuelas. Buenos Aires: Paidós Educador.

JOHNSON, D.; Johnson, R. y Holubec, E. (1999). El Aprendizaje cooperativo en el aula. Buenos Aires: Paidós.

LABARRERE, A. (2016). Zona de Desarrollo Próximo como eje del desarrollo de los estudiantes: de la ayuda a la colaboración. Summa Psicológica. 13 (1) 45 - 56. Disponible: https://dialnet.unirioja.es/servlet/articulo?codigo $=5585077$

LEÓN, B.: Felipe. E.; Iglesias, D. y Latas, C. (2011). El aprendizaje cooperativo en la formación inicial del profesorado de Educación Secundaria. Revista de Educación. (354) 715 - 729. 
Disponible: $\quad$ http://www.educacionyfp.gob.es/revista-de-educacion/numeros-revistaeducacion/numeros-anteriores/2011/re354/re354-28.html

LÓPEZ, R. P. (2015). Posibilidades y limitaciones del aprendizaje cooperativo en el EEES: análisis de una experiencia en el aula de inglés. Porte Linguarum (24) 163 - 177. doi: $\underline{\text { 10.30827/Digibug. } 53868}$

MADRID, J. C.; Arellano, M.; Jara, R.; Merino, C. y Balocchi, E. (2013). El aprendizaje cooperativo en la comprensión del contenido "disoluciones". Un estudio piloto. Revista Educación Química., (24) 471 - 479. doi: https://doi.org/10.1016/S0187893X(13)72515-1

MARTÍNEZ, M. (1998). La investigación cualitativa etnográfica en educación: Manual teórico - práctico. (3era. edic.) México: Trillas.

MUÑOZ, Y. y Cordero, N. (2017). El aprendizaje cooperativo como metodología para el desarrollo de una escuela inclusiva. Revista Brasilera de Psicología Educativa, 19 (1) 149 - 162. doi: 10.30715/rbpe.v19.n1.2017.10826

MEDRANO, C. (1995). La interacción entre compañeros: el conflicto sociocognitivo, el aprendizaje cooperativo y la tutoría entre iguales. Revista Interuniversitaria de formación del profesorado, (23) 177 - $186 . \quad$ Disponible: https://dialnet.unirioja.es/servlet/articulo?codigo $=117874$

MORALES, M. L.; García, M. O.; Torres, R. A. y Lebrija, T. A. (2018). Habilidades cognitivas a través de la estrategia de aprendizaje cooperativo y perfeccionamiento epistemológico en matemáticas de estudiantes de primer año de universidad. Formación Universitaria, 11 (2) 45 - 56. DOI: http://dx.doi.org/10.4067/S0718-50062018000200045

MOYA, P. y Zariquiey, F. (2008). El aprendizaje cooperativo: una herramienta para la convivencia. En Torrego, Juan. (ed.). El plan de convivencia. Fundamentos y recursos para su elaboración y desarrollo. (pp. 267 - 314). Madrid: Alianza Editorial.

MURILlO, J.; Hernández, R. y Martínez, C. (2016). ¿Qué ocurre en las aulas donde los niños y niñas no aprenden? Estudio cualitativo de aulas ineficaces en Iberoamérica. Perfiles $\begin{array}{llllll}\text { Educativos. } & 38 & (151) & 55 & - & 70 .\end{array}$ http://www.scielo.org. $m x /$ scielo.php? script $=$ sci_arttext\&pid=S0185$\underline{26982016000100055}$

OBERTO, T. (2014). El aprendizaje cooperativo como herramienta para la educación universitaria. Revista Educación en Valores., 1 (21) 58 - 69. Disponible: https://dialnet.unirioja.es/servlet/articulo?codigo $=7022175$

PAMPLONA, R. J.; Cuesta, S. J. y Cano V. V. (2019). Estrategias de enseñanza del docente en las áreas básicas: una mirada al aprendizaje escolar. Revista Eleuthera., (21) 13 - 33. doi: 10.17151/eleu.2019.21.2.

PEÑA, J. D. (2010). El aprendizaje cooperativo y las competencias. Revista d'Innovació

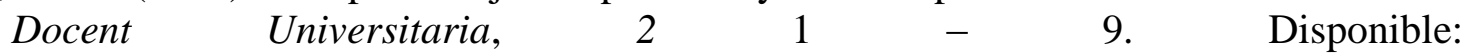
https://dialnet.unirioja.es/servlet/articulo?codigo $=5053550$

PÉREZ, S. G. (1994). Investigación Cualitativa. Retos e Interrogantes. (Tomo II). Madrid. La Muralla.

PÉREZ de A. A.; Escolano, E.; Pascual S. M.; Lucas, B. y Sastre, S. (2015). Metacognición en un proceso de aprendizaje autónomo y cooperativo en el aula universitaria. Contexto $\begin{array}{lllll}\text { Educativo, } & \text { (18) } & 95 & - & 108 .\end{array}$ https://publicaciones.unirioja.es/ojs/index.php/contextos/article/view/2576

REVELO, S. O.; Collazos, O. C. y Jiménez, T. J. (2017). El trabajo colaborativo como estrategia didáctica para la enseñanza/aprendizaje de la programación: una revisión sistemática de 
la literatura. TecnoLógicas, 21 (41) $115 \quad-\quad$ 134. Disponible de http://www.scielo.org.co/pdf/teclo/v2 $\ln 41 / v 2 \ln 41 a 08 . p d f$

RODRÍGUEZ, G. G.; Gil, F. J. y García, J. E. (1996). Metodología de la Investigación Cualitativa. Málaga: Aljibe, Ediciones.

SLAVIN, R. (1999). Aprendizaje Cooperativo. Teoría, Investigación y Práctica. Buenos Aires: Aique Grupo Editor S.A.

TORRADO, A. D.; Manrique, H. E. y Ayala, P. J. (2016). La tutoría entre pares: una estrategia de enseñanza y aprendizaje de histología en la Universidad Industrial de Santander. Revista de los estudiantes de Medicina de la Universidad Industrial de Santander, 29 (1). 71 - 75. Disponible: http://www.scielo.org.co/pdf/muis/v29n1/v29n1a09.pdf

TRUJILlo, F. (2002). Aprendizaje Cooperativo para la enseñanza de la lengua. Revista Publicaciones de la Facultad de Educación y Humanidades - Campus de Melilla. (32) 147 - $\quad$ 162. Disponible: $\quad$ https://fernandotrujillo.es/wpcontent/uploads/2010/05/cooperacion.pdf

UZCÁTEGUI, P. R.; Guzmán, G. Wendy y Bravo, J. L. (2018). El proceso migratorio y el vaciamiento de la institucionalidad educativa escolar en Venezuela. Revista Electrónica de Investigación Educativa Sonorense, Número Especial. 20 - 49. Disponible: https://rediesonorense.wordpress.com/

VAILLANT, D. y Manso, J. (2019). Orientaciones para la formación docente y el trabajo en el aula. Aprendizaje colaborativo. [Libro en Línea]. SUMMA: Santiago. Disponible: https://www.summaedu.org/lab-ed/

VILLARRUBIA, S. (2016). El aprendizaje cooperativo como metodología para la atención a la diversidad. (Tesis de Maestría). Universidad Internacional de la Rioja. Barcelona, España.

VYGOTSKY, L. (1979). El desarrollo de los procesos Superiores. Barcelona: Critica.

WOODS, P. (1987). La escuela por dentro: la etnografía en la investigación educativa. Madrid: Paidós Ibérica S.A.

ZURITA, M. (2020). El aprendizaje cooperativo y el desarrollo de las habilidades cognitivas. Revista Educare. 24 (1) 51 - 74. Disponible: https://revistas.investigacionupelipb.com/index.php/educare/article/view/1226

Autores

Gilberto Aranguren Peraza. Profesor en Ciencias Naturales con Mención de Química (UPEL - IPC), Maestría y Doctorado en Ciencias de la Educación (UNESR), Post Doctorado en Filosofía y Educación (UCV). Profesor en Ciencias Naturales en Educación Media y Profesor de UNESR y otras Universidades. gilberap@gmail.com https://orcid.org/0000-0003-1480-9401

Marisol Sellet Zurita Aguilera. Licenciada en Educación Mención Planificación (Universidad Central de Venezuela)Especialización en Evaluación de los Aprendizaje (UPEL)Maestría en Ciencias de la Educación con mención en Psicología de los Procesos de Aula Doctora en innovaciones Educativas (UNEFA). Profesora Adscrita a la Universidad Nacional Experimental de las Fuerzas Armadas (UNEFA) margasam4@gmail.com https://orcid.org/0000-0002-2347-2575

\section{Como citar el artículo:}

Aranguren Peraza, Gilberto; Zurita Aguilera, Marisol Sellet. Impacto de la aplicación del aprendizaje cooperativo en aulas de Educación Básica. Revista Paradigma Vol. XLII, Nro. 2, Diciembre de 2021 / 350 - 374.

DOI: https://doi.org/10.37618/PARADIGMA.1011-2251.2021.p350-374.id1081 
Gilberto Natividad Aranguren Peraza; Marisol Sellet Zurita Aguilera

Revista Paradigma Vol. XLII, Nro. 2, diciembre de 2021 / 350 - 374 